\title{
手性双金属催化剂及其在不对称催化反应中的应用
}

\author{
李昆杨国强刘媛媛张万斌* \\ (上海交通大学化学化工学院 上海 200240)
}

\begin{abstract}
摘要 多金属催化过程是生命体实现新陈代谢的主要方式之一, 在自然界广泛存在. 多金属催化剂相比于单金属催化 剂, 具有更加高的反应活性和对映选择性，近年来其设计与应用已经成为有机化学的研究热点之一. 综述了近年来不 同种类的具有代表性的双金属催化剂的研究进展，包括双杂金属和双核同金属，介绍了双金属催化剂的反应类型、反 应机理以及发展现状, 提出了今后的研究重点与发展趋势.
\end{abstract}

关键词 不对称催化; 多金属; 双核同金属催化剂; 双杂金属催化剂

\section{Chiral Polymetallic Catalysts and Their Applications in Asymmetric Catalysis}

\author{
Li, Kun Yang, Guoqiang Liu, Yuanyuan Zhang, Wanbin* \\ (School of Chemistry and Chemical Engineering, Shanghai Jiaotong University, Shanghai 200240)
}

\begin{abstract}
Polymetallic catalytic process is the main metabolic way of life, which is widely observed in nature. Compared to the mono-metal catalysts, the designs and applications of polymetallic catalysts with high reactivity and enantioselectivity have been a hot research topic in organic chemistry recently. This review covers the recent research process on representative types of bimetallic catalysts, including hetero-bimetallic catalysts and dinuclear homometal catalysts, their reaction mechanisms and developments. Moreover, potentially development of this area is also discussed.
\end{abstract}

Keywords asymmetric catalysis; polymetallic; dinuclear homometal catalyst; hetero-bimetallic catalyst

生命体的新陈代谢极大程度地依赖于各种各样的 酶, 这些酶具有非常高的催化活性, 为生命体的能量和 物质的吸收与释放提供了简便快捷高效的传递方式, 而 这些酶又以多金属中心酶为主. 双核杂金属 $(\mathrm{Cu}, \mathrm{Zn})$ 酶 SOD (superoxide disproportionation)可以分解自由基氧, 减缓衰老; 双核同金属 $(\mathrm{Fe}$ )酶 MMO (Methane monooxygenase)可以在温和条件下用氧气氧化甲烷为甲醇, 这些自然的杰作提示了化学家们: 多金属催化剂可以实 现单金属催化剂所不能实现的反应, 或可以比单金属催 化剂的催化性能更好. 然而如何设计合理的多金属催化 剂才能实现化学催化的突破是一个摆在化学家面前的 难题. 最为简单的便是从多金属酶的结构出发, 模仿其 结构, 从而实现较小分子催化剂的酶水平催化 ${ }^{[1]}$. 当然, 化学家们也可以通过分析来设计自然界中所不存在的
多金属催化剂结构模型, 这是更具有挑战的研究工作.

多金属催化剂可按金属种类分为同金属催化剂，双 杂金属催化剂和多杂金属催化剂, 也可按金属的个数分 类为双核金属催化剂和多核金属催化剂. 两种分类方法 也可以相互交叉进行分类: 双核同金属、双核杂金属、 多核同金属、多核双金属、多核杂金属(图 1) $)^{[1]}$.

双金属催化剂中金属原子与配体的配位方式可分 为以下两类(图 2): $\mathrm{a}$ 类为两金属原子有共用的配位原子 或基本结构 $\mathrm{B}$, 如 $\mathrm{O}, \mathrm{S}, \mathrm{C} \equiv \mathrm{C}$ 的两个 $\pi$ 键、氮氮键的两 氮原子、氧氧键的两氧原子等; $b$ 类为无共用的配位原子 或基本结构 $\mathrm{B}$, 只是用一些简单的 “化学链” 来将两个 配体链接起来. 可根据需要按照下面的配体模型来设计 所需要的配体, 通过对 B 和 L 及各 “化学链” 的修饰进 而实现所需研究的催化反应.

\footnotetext{
* E-mail: wanbin@sjtu.edu.cn

Received January 14, 2013; revised February 14, 2013; published online February 22, 2013. sion of Shanghai Municipality (No. 09JC1407800).

国家自然科学基金(Nos. 20972095, 21172143, 21232004)和上海市科学技术委员会(No. 09JC1407800)资助项目.
}

Project supported by the National Natural Science Foundation of China (Nos. 20972095, 21172143 and 21232004) and the Science and Technology Commis- 


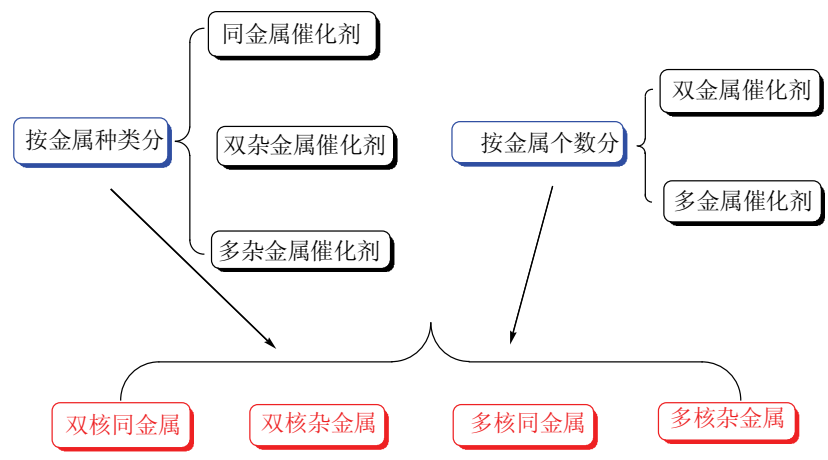

图 1 多金属催化剂的分类

Figure 1 Classification of polymetallic catalysts
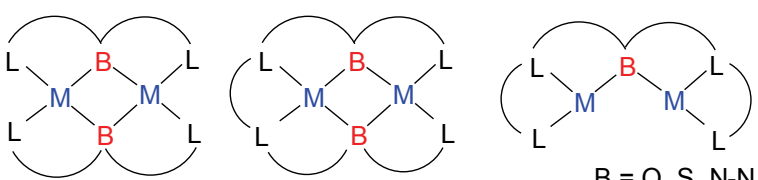

$\mathrm{B}=\mathrm{O}, \mathrm{S}, \mathrm{N}-\mathrm{N}$ $\mathrm{O}-\mathrm{O}, \mathrm{N}-\mathrm{O}$<smiles>[M]1CCCCCCCI=[Al]1</smiles>
配位方式(a)

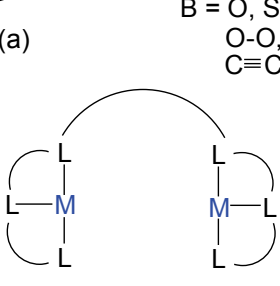

配位方式(b)

图 2 多金属催化剂的分类

Figure 2 Classification of polymetallic catalysts

目前的双金属催化剂的催化反应方式主要分为以 下五类(图 3), 依次为: (a)两种反应物 $S, R$ 与两金属原子 分别配位, 然后反应生成 $\mathrm{S}-\mathrm{R}$; (b) 底物 $\mathrm{S}$ 与两金属原子 同时配位，另一底物 $\mathrm{R}$ 直接进攻 $\mathrm{S}$ 生成 $\mathrm{S}-\mathrm{R} ;(\mathrm{c})$ 底物 $\mathrm{S}$ 与两金属原子同时配位, $\mathrm{R}$ 与一金属原子配位, 然后反 应生成 S-R; (d) 只有底物 $\mathrm{S}$ 与一金属原子配位, 另一底 物 $\mathrm{R}$ 直接进攻 $\mathrm{S}$ 生成 $\mathrm{S}-\mathrm{R}$, 而另一金属原子 $\mathrm{M}^{2}$ 起到了 调节 $\mathrm{M}^{1}$ 电子性能的作用; (e)两种反应物 $\mathrm{S}, \mathrm{R}$ 与同一金 属原子配位, 然后反应生成 $\mathrm{S}-\mathrm{R}$, 而另一金属原子 $\mathrm{M}^{2}$ 起到了调节 $\mathrm{M}^{1}$ 电子性能的作用.

近年来, 从事手性催化的化学家们注意到了多金属 催化剂的独特性能, 他们已经开发了一系列的多金属催 化剂并将之应用于各类不对称催化反应，这些多金属手 性催化剂主要有双核同金属催化剂和双杂金属催化剂. 以下所指的双金属催化剂是指多个金属原子合作共同 催化反应的催化剂. 目前也有一些被报道的具有两个以 上金属原子的催化剂, 但各金属原子各自催化反应, 不 属于本文综述的范畴.

\section{1 双杂金属催化剂的发展现状}

手性双杂金属催化剂的开发以日本化学家 Shibasaki 最为著名, Shibasaki 的手性双杂金属催化

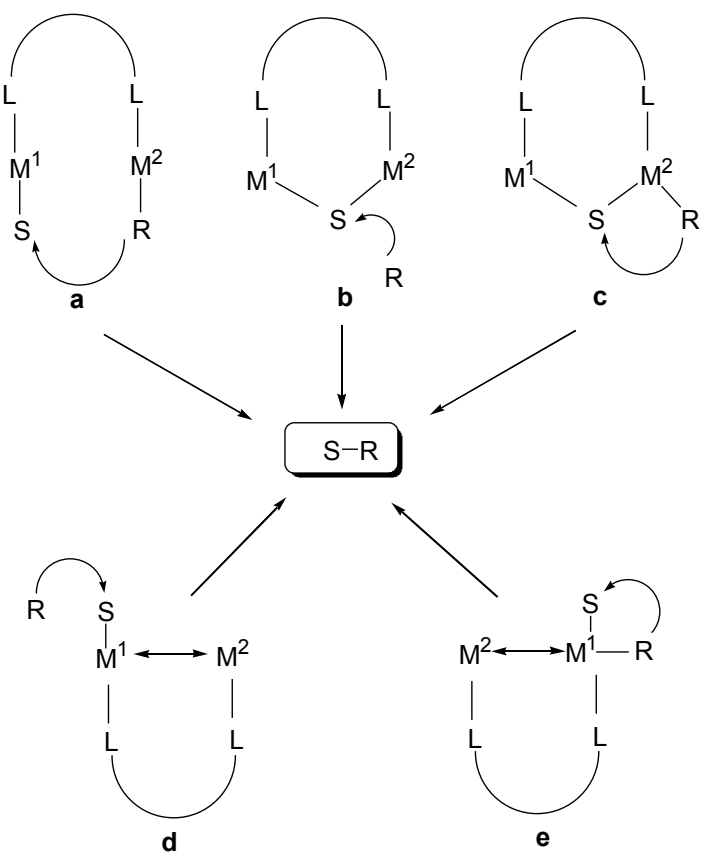

图 3 双金属催化剂的配位方式

Figure 3 Catalysis Types of Bimetallic Catalysts

剂 $[2,3]$ 可以分为双核杂金属双功能催化剂, 多核杂金属 双功能催化剂(图 4). 催化剂 $\mathbf{4 c}$ 虽然两金属原子都为 $\mathrm{Ni}$, 但 $\mathrm{Ni}$ 所处环境不同, 功能不同, 权且归于此类. 这些催 化剂中的一个金属原子用作 Lewis 酸活化一个羰基，另 一个金属原子与氧作用使得氧的 Brønsted 碱性增强. 它 们在硝基烷烃对醛 ${ }^{[2 b]}, \alpha, \beta$-不饱和羰基化合物的亲核加 成反应 ${ }^{[2 \mathrm{c}]}$, 酮对醛的 Aldol 反应 ${ }^{[2 \mathrm{~d}]}$, 环氧化 $\alpha, \beta$-不饱和 羰基化合物 ${ }^{[2 \mathrm{e}]}$, 膦氢键加成亚胺 ${ }^{[2 \mathrm{f}]}$ 、醛 ${ }^{[2 \mathrm{~g}]}$ 等反应 (Scheme 1)中都取得了很好的对映选择性.

Shibasaki 双杂金属催化剂的催化机理：以硝基烷 烃对醛的加成为例, 催化剂 $\mathbf{1 a}$ 与 1 equiv. 的 $\mathrm{LiOH}$ 生成 活性催化剂 $\mathbf{6}$, 氢氧根与 $\mathrm{La}$ 配位, 然后与一分子硝基烷 烃反应生成一分子水，硝基烷基负离子与两种金属原子 配位得到中间体 7, 醛基与 $\mathrm{La}$ 配位后，硝基烷基负离子 
<smiles>[R]c1ccc2c(-c3c(CC)cc4ccc([R])cc4c3O)c(O)ccc2c1</smiles>

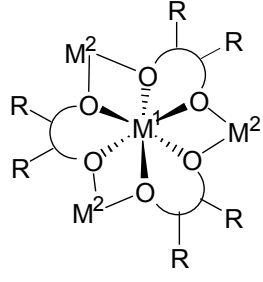

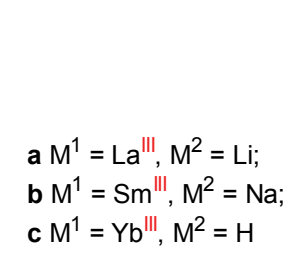

多核杂金属双官能催化剂

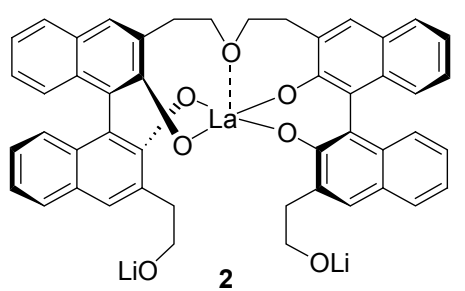<smiles>COc1ccc2ccccc2c1-c1c(OCl)ccc2ccccc12</smiles>

3

$$
\mathrm{M}^{3}=\mathrm{Al}^{\mathrm{III}}, \mathrm{Sc}^{\mathrm{III}}
$$

双核杂金属双官能催化剂

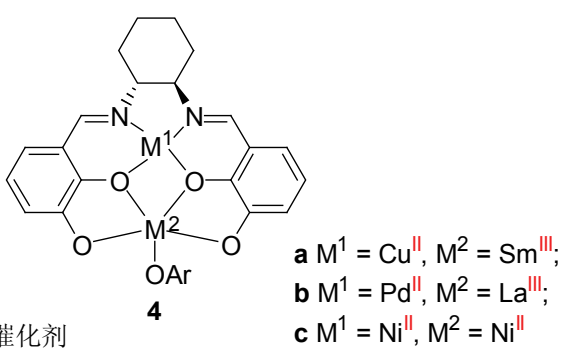

图 4 Shibasaki 双杂金属催化剂

Figure 4 Shibasaki hetero-bimetallic catalysts

$$
\text { 至. }
$$

\section{Scheme 1}

进攻羰基碳，所得醇负离子相当于氢氧根的作用，再与 硝基烷烃反应生成一分子产物, 实现催化循环(Scheme 2). 硫醇对 $\alpha, \beta$-不饱和羰基化合物的 Micheal 加成反应 也是类似的机理.
2007 年, Shibasaki 小组 $\left.{ }^{[3]}\right]$ 报道了手性席夫碱双金属 催化剂配体 4, 并将之与金属原子 $\mathrm{Cu}$ 和 $\mathrm{Sm}$ 配位形成手 性催化剂 $4 \mathbf{a}$, 用于催化亚胺的 Henry 反应时得到了很好 的对映选择性和顺式选择性(Scheme 3).<smiles></smiles><smiles>CC(CBr)C(C)C1CCCO1</smiles><smiles></smiles>

6 LLB-II

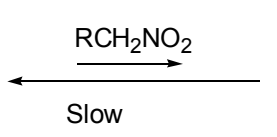

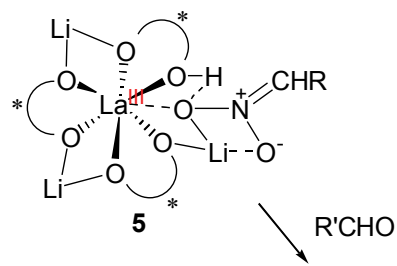

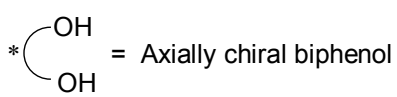<smiles>[R]C(O)C([R])[N+](=O)[O-]</smiles>

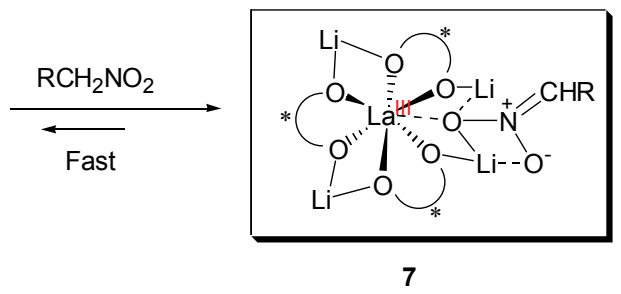

Scheme 2 


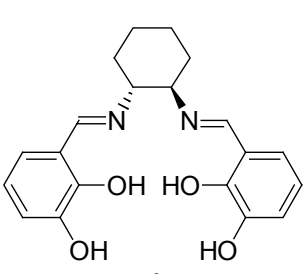

4<smiles>[R]C[N+](=O)[O-]</smiles>

yield $>66 \%$

dr $>20: 1$, ee $83 \% \sim 97 \%$

\section{Scheme 3}

2008 年, 该小组 ${ }^{[3 b]}$ 将催化剂 $\mathbf{4 a}$ 用于催化底物 $\mathbf{8}$ 与 亚胺的 Henry 反应时发现催化效果很差, 于是他们将配 体的手性骨架换为联萗, 并且进行了一系列的金属篮选 工作，发现两金属原子都是 $\mathrm{Ni}$ 时，催化剂 9 催化效果几 乎完美, 产率为 $95 \%$, 对映选择性为 $98 \%$, 令人惊喜的 是得到了反式选择性的产物, 且选择性为 $91: 9$, 这是 首例报道的反式选择性的 Henry 反应(Eq. 1). 紧接着该 小组 ${ }^{[3 c]}$ 又报道了配体 4 与 $\mathrm{Pd}$ 和 $\mathrm{La}$ 配位后得到的催化剂 催化 Henry 反应，也选择性地得到反式产物.
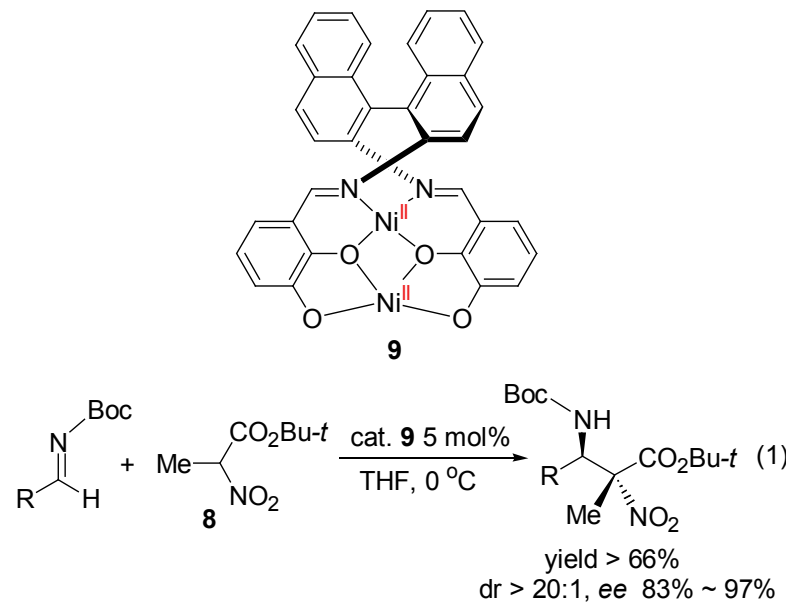

最近, Shibasaki 等 ${ }^{[4]}$ 设计了配体 $\mathbf{1 0}$, 当将 $\mathbf{1 0 a}$ 与 $\mathrm{Gd}$ 配位催化酮的氰硅化反应时(Scheme 4), 发现催化效果 非常好. 于是该小组对催化机理进行了研究, 质谱表明 催化反应的中间体为：两金属原子中心与氰基配位后, 其中一金属原子与酮羰基配位，与另一金属原子配位的 氰基进攻羰基碳即得产物，这说明该催化反应的活性催 化剂也是双金属催化剂，而且是双核同金属催化剂.

与此同时，铜催化的有机锌试剂对缺电子不饱和双 键的 1,4-共轭加成反应是另一类主要的手性双杂金属催
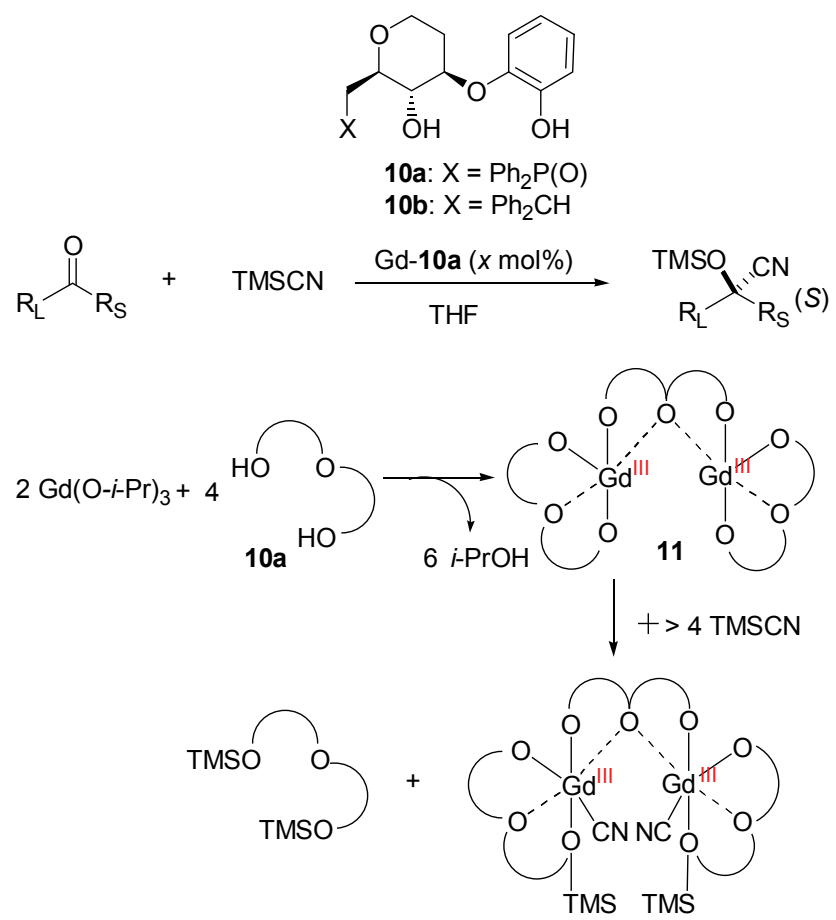

$\left[\mathrm{M}^{+}-\mathrm{CN}\right]=1753.4$ (observed by ESI-MS)

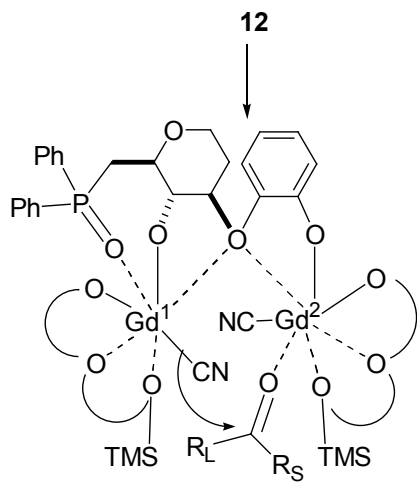

13

Scheme 4

化反应. 化学家们已经开发出了非常高效的手性配体应 用于铜催化的该类反应，其中亚磷酰胺类配体具有很高 的化学选择性和立体选择性 ${ }^{[5]}$.

对于铜催化的有机锌进行的 1,4-加成反应, Feringa 等 ${ }^{[6]}$ 提出了反应的机理(Scheme 5): 首先铜盐和 手性亚膦酰胺(酯)类配体形成类似二聚体的一价铜-手 性亚膦酰胺配体络合物 $\mathbf{1 4}$, 其中两个 $\mathrm{Cu}$ 原子形成一个 混合的三角/四面体构型. 随后烷基从 $\mathrm{Zn}$ 试剂转移到 $\mathrm{Cu}$ 上形成络合物 15 . 一价铜与环己烯酮通过 $\pi$ 电子配位, 二价锌作为路易斯酸与羰基结合，两者通过另一个铜原 子连接形成中间体络合物 16 , 经过氧化加成形成 $\mathrm{Cu}(\mathrm{III})$ 中间体 17 , 之后通过还原消除形成具有活性的催 化剂 14 和有机锌化合物 18 , 该步骤为反应的决速步. 形成的 14 参与到下一个催化循环中, 而产生的有机锌 
化合物 18 可以通过质子化形成简单的共轭加成产物, 或者通过不同的亲电基团形成不同的产物.

1996 年, Feringa 等首先报道了一类新型的以 BINOL 和胺基为骨架的亚膦酰胺类配体, 并在随后将 改进的配体 19 用于铜催化的有机锌为亲核试剂的 1,4加成反应, 首次成功得到了 $90 \%$ 以上的对映选择性
(Scheme 6 $)^{[7]}$. 之后通过改变芳基和胺基衍生出的一系 列的亚磷酰胺类配体, 对不同类型的环烯酮和开链烯酮 底物的 1,4-共轭加成反应取得了极好的效果 ${ }^{[8 \sim 10]}$. 另一 方面, 随着对于 1,4-共轭加成反应研究的不断深入，一 些其它类型的配体同样被开发出来, 它们经历类似的双 金属催化过程并且具有很好的效果 ${ }^{[11 ~ 13]}$ (图 5).
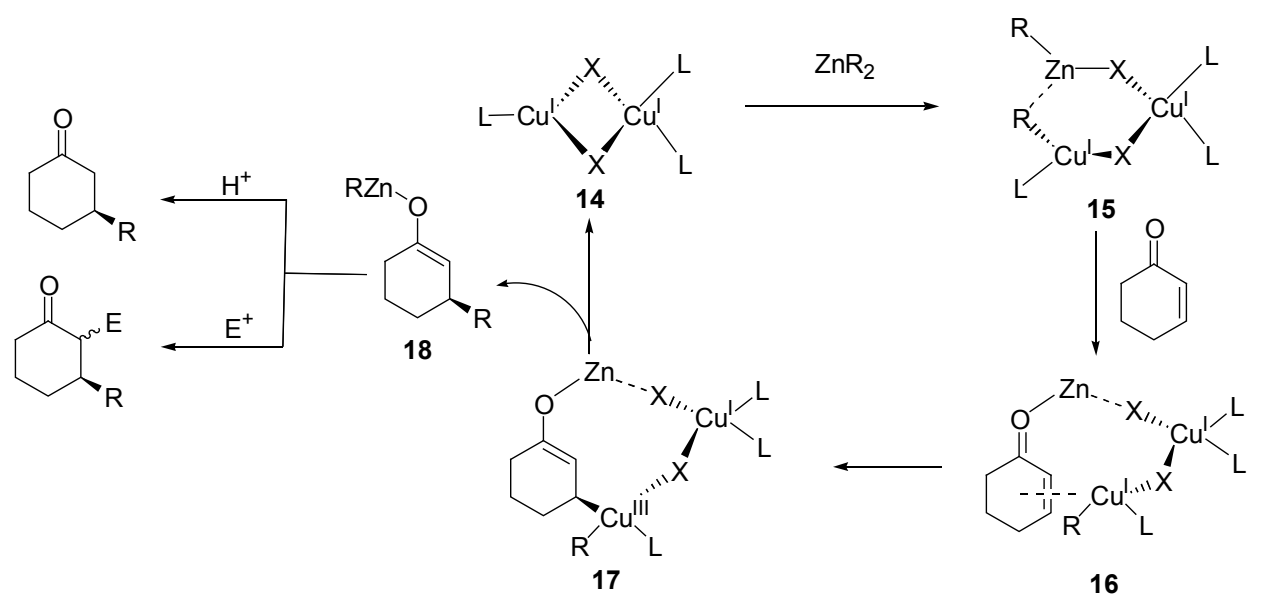

Scheme 5

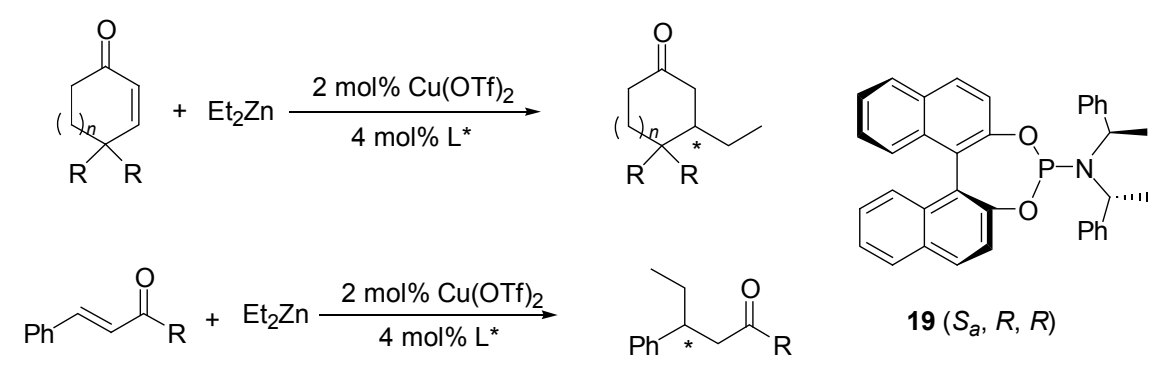

Scheme 6

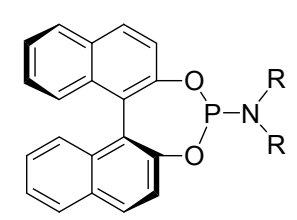

$\mathrm{R}: \mathrm{Me}, i-\mathrm{Pr}$ Monophose

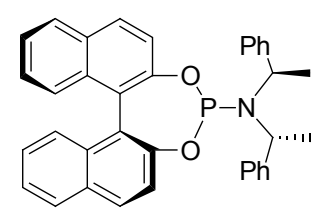

$19\left(S_{a}, R, R\right)$

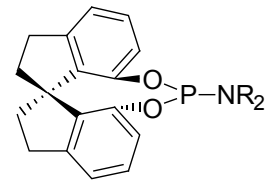

20a $\mathrm{R}=(R)-\mathrm{CH}(\mathrm{Ph}) \mathrm{CH}_{3}$ 20b $\mathrm{R}=(\mathrm{S})-\mathrm{CH}(\mathrm{Ph}) \mathrm{CH}_{3}$<smiles>[R]c1cc([R])c2op(N([R])C([R])C)oc3c([R])cc([R])cc3c2c1</smiles>

$$
\begin{aligned}
& \text { 21a } R^{1}=H, R^{2}=P h \\
& \text { 21b } R^{1}=M e, R^{2}=P h \\
& \text { 21c } R^{1}=M e, R^{2}=2-N a p
\end{aligned}
$$<smiles>CC(C)(C1=NC(c2ccccc2)CO1)c1nnc(C(C)(C)C2=NC(c3ccccc3)CO2)c2ccccc12</smiles>

25

$\mathrm{R}: \mathrm{a}(\mathrm{H}),($ b) $\mathrm{Me},(\mathrm{c}) \mathrm{Et}$, (d) Br, (e) Allyl

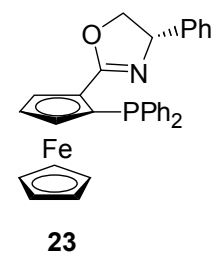<smiles></smiles>

图 5 不同类型 1,4-共轭加成反应配体

Figure 5 Ligands for 1,4-conjugate addition reactions 


\section{2 双核同金属催化剂的发展现状}

相对于双杂金属催化剂的研究主要由 Shibasaki 小 组进行, 双核同金属催化剂的开发则受到更多研究小组 的关注. 较为活跃的小组有 Jacobsen, Trost, Maruoka 等.

Trost 小组 ${ }^{[14]}$ 的半冠醚氨基醇类双金属锌催化剂(图 6)与 Shibasaki 的双杂金属催化剂类似, 两金属原子的作 用不同, 一个为 Lewis 酸, 一个为 Brønsted 碱. 因此它们 的应用也相似。同时 Trost 小组还将该催化剂应用于 Friedel-Crafts-Micheal 加成反应 ${ }^{[14 \mathrm{e}]}$ 和二醇的去对称化反 应 $^{[14 c]}$ (Scheme 7).

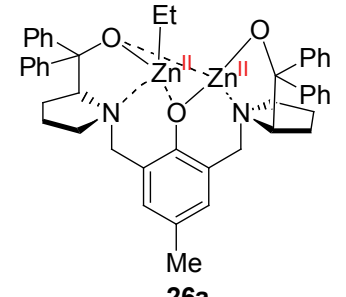

$26 a$

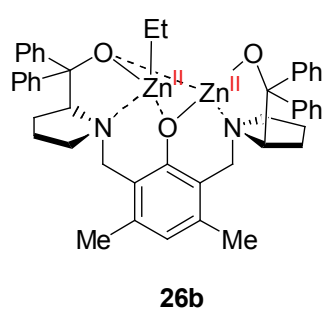

图 6 Trost 手性双金属锌催化剂

Figure 6 Trost chiral dizinc catalysts

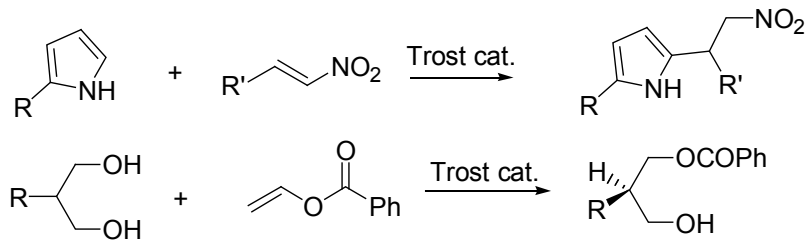

\section{Scheme 7}

以 Aldol 反应举例说明 Trost 半冠醚氨基醇类双金 属锌催化剂的催化机理 ${ }^{[14 \mathrm{a}]}$. 该催化剂都是原位生成, 当配体与 2 equiv.的二乙基锌反应时，离去三分子的乙 烷, 得到的催化剂如 $26 \mathrm{a}$ 或 $26 \mathrm{~b}$, 仍有一个乙基与锌配 位，两个锌离子均采取 $\mathrm{sp}^{3}$ 杂化的四面体配位形式. 当 加入底物酮与醛后，乙基与酮反应离去乙烷. 此时酮以 烯醇式与配体中一锌离子配位, 另一锌离子与醛配位 后, 酮与醛都被活化, 发生反应得到 $\beta$-酮醇负离子, 空 出一个配位点. 然后另一分子底物酮与之配位, 醇负离 子夺去该酮的 $\alpha$ 氢生成产物离去, 从而完成了一次催化 循环(Scheme 8).

与 Trost 双金属锌的人为设计相比, Jacobsen 双席夫 碱双金属催化剂则是偶然发现. Jacobsen 在进行席夫碱 Co 配合物催化环氧化合物开环反应的机理研究时发现, 反应速率与催化剂量的平方成正比, 这就说明催化的中 间态是双催化剂协同作用的，于是 Jacobsen 将两单独的 催化剂连接起来, 结果发现催化剂的催化效率提高了很 多. Jacobsen 双金属催化剂有两类 31 和 32(图 7).

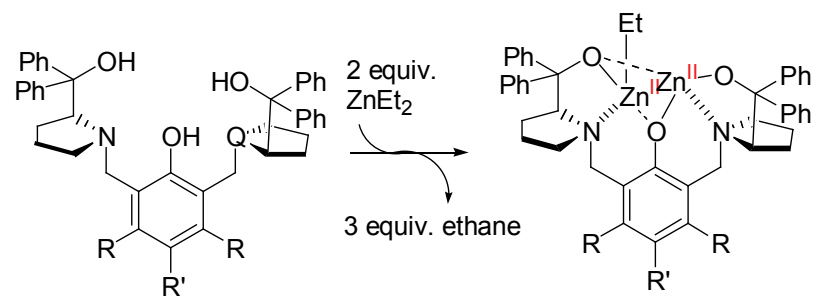

26a: $\mathrm{R}=\mathrm{H}, \mathrm{R}^{1}=\mathrm{CH}_{3}$

26b: $\mathrm{R}=\mathrm{CH}_{3}, \mathrm{R}^{1}=\mathrm{H}$

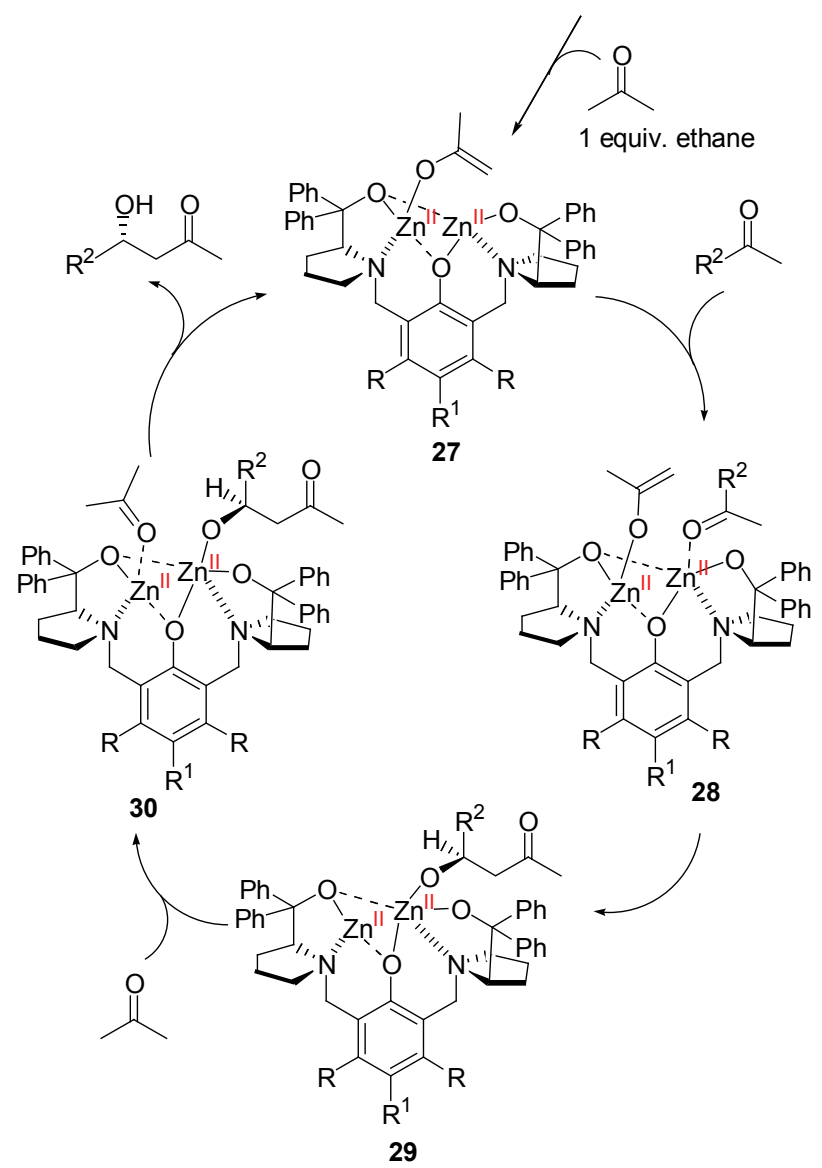

Scheme 8

31-Co 主要就用于环氧化合物的开环反应 ${ }^{[15 \mathrm{a}]}$ (Scheme 9), ee 值都很高, 且催化剂效率很高，最低用量 达到了 $0.0004 \mathrm{~mol} \%^{[15 b]}$, 这说明该催化剂在工业化应用 中的潜力是非常大的.

31-Al 则应用于 $\alpha, \beta$-不饱和酰胺化合物与氰硅烷的 加成反应 ${ }^{[15 c]}$ (Scheme 9), 催化剂用量也较低, 且对映选 择性大多大于 $90 \%$.

三齿席夫碱配体与 $\mathrm{Cr}$ 的配合物 32 作为手性催化剂 应用于醛的分子间和分子内杂 ene 反应，取得了很好的 结果，催化剂的活性和对映选择性都很高. Jacobsen 等 ${ }^{[16]}$ 设计了一系列复杂的可发生分子内 ene 反应的底 物, 32 催化这些底物发生反应得到了非常有用的五元和 六元杂环化合物(Scheme 10). 


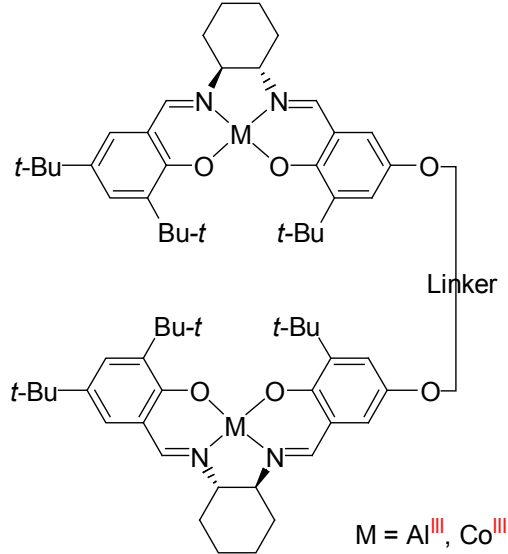

31

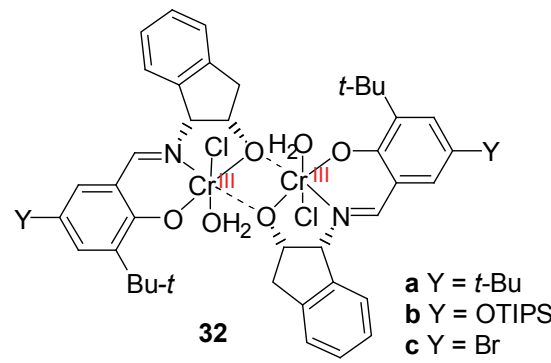

32

图 7 Jacobsen 双金属催化剂

Figure 7 Jacobsen chiral bimetallic catalysts

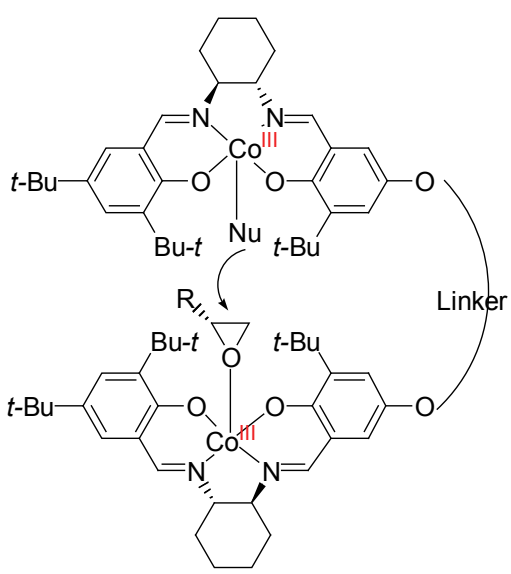

31-Co
$\mathrm{O}_{31-\mathrm{Co}}^{\mathrm{R}^{2}} \underset{\mathrm{HO}}{\stackrel{\mathrm{NuH}}{\mathrm{R}^{1}}} \overbrace{\mathrm{Nu}}^{\mathrm{R}^{2}}$

不对称催化环氧化合物开环反应

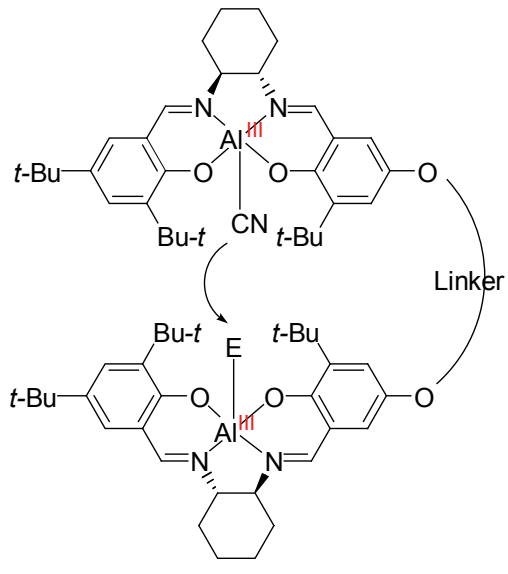

31-Al

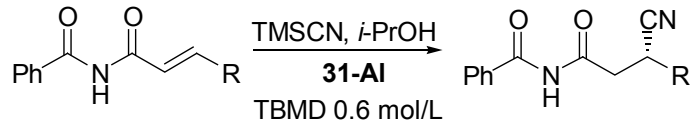

$50{ }^{\circ} \mathrm{C}$

\section{Scheme 9}

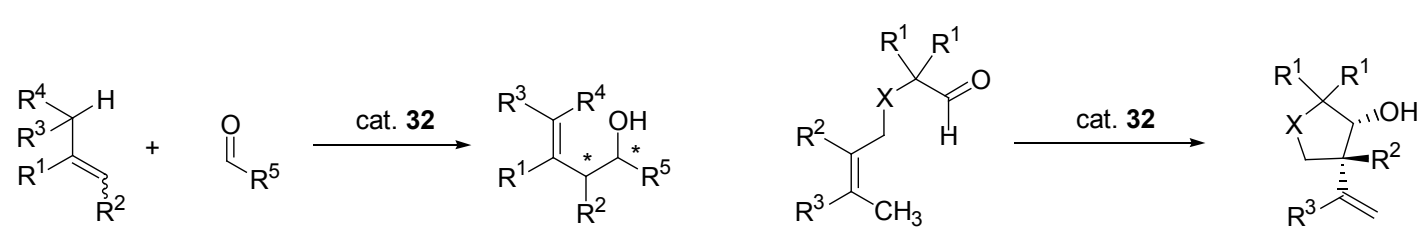

Scheme 10

最近, Hong 等 ${ }^{[17]}$ 报道了一例通过氢键作用将 Jacobsen 席夫碱-Co 催化剂自组装为双金属催化剂 33, 使用该催化剂催化 Henry 反应时, 取得了 $95 \%$ 的对映选 择性，与单金属催化剂 34 的催化效果(11\%产率，55\% $e e$ )形成了鲜明对比(Scheme 11), 催化反应速率与单体 催化剂量(即氢键组装催化剂量的两倍)的平方成正比, 证明了双金属催化剂的协同催化机理, 具体催化机理正
在研究中.

2003 年, Maruoka 小组 ${ }^{[18]}$ 报道了氧相连的 Binol-Ti 双金属钠催化剂 35 (图 8), 该催化剂具有双活化醛基的 作用，将该催化剂应用于有挑战性的醛基化合物的反应 时发现, 双金属钛催化剂可以催化单金属钛催化效果很 差的反应，且催化效果很好(Scheme 12). 


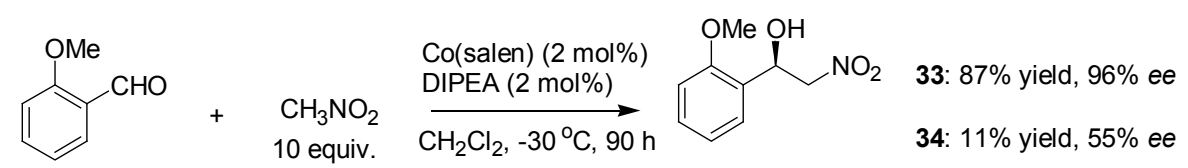

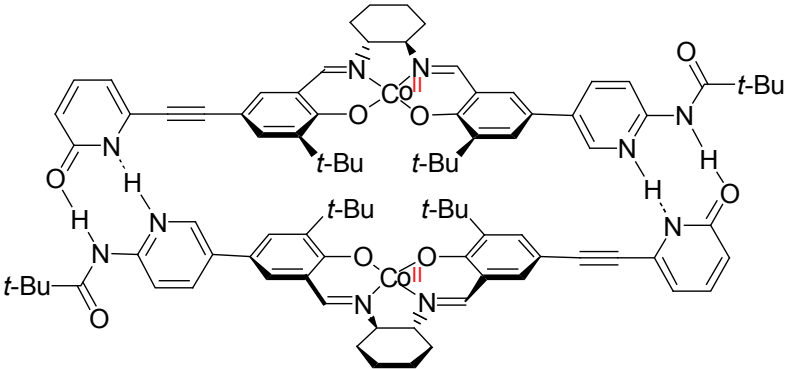

33

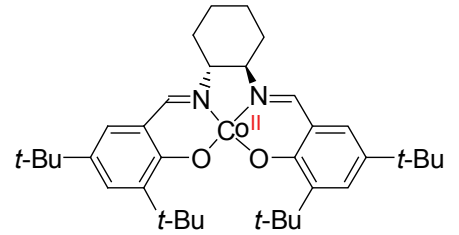

34

Scheme 11

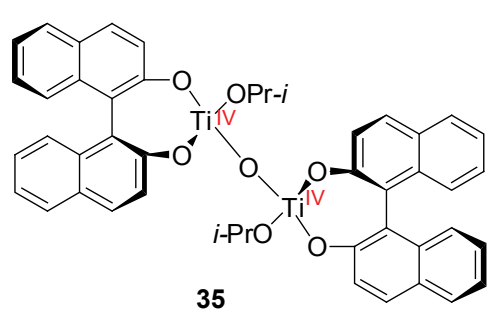

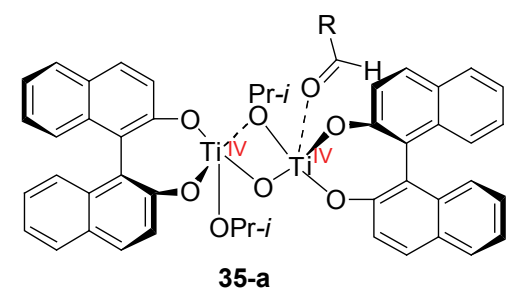

35-a

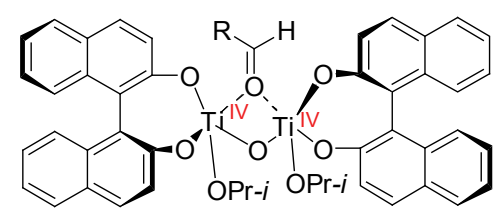

35-b

图 8 Maruoka 手性双 BINOL-Ti 催化剂

Figure 8 Maruoka Chiral BisBINOL-Ti Catalyst

yield $60 \% \sim 90 \%)$

$\int_{\mathrm{CHO}}^{\mathrm{Me}}+\mathrm{N}_{2} \mathrm{CHCO}_{2} \mathrm{R} \stackrel{35(10 \mathrm{~mol} \%)}{\longrightarrow}$ up to $94 \%$ ee and 82 yield

$$
\begin{aligned}
& \text { 1) } 35(10 \mathrm{~mol} \%) \\
& \text { 2) } \mathrm{NaBH}_{4}
\end{aligned}
$$

\section{Scheme 12}

North 等 ${ }^{[19 a]}$ 于 1999 年报道了 Salen 双钛 36 不对称 催化 Strecker 反应, 之后的机理研究也表明该催化过程 是一个双金属过程 ${ }^{[19 b]}$. 最近, 丁奎岭小组 ${ }^{[19 c]}$ 发展了两 个 Salen 配体连接起来的催化剂 37 , 大大地改进了催化 剂的催化活性和选择性(Scheme 13).

另外, Kobayashi 等 ${ }^{[20]}$ 也报道了一例类似的催化剂 为双金属铌催化剂 38(图 9), 并且将之应用于亚胺的 Mukaiyama 的反应.
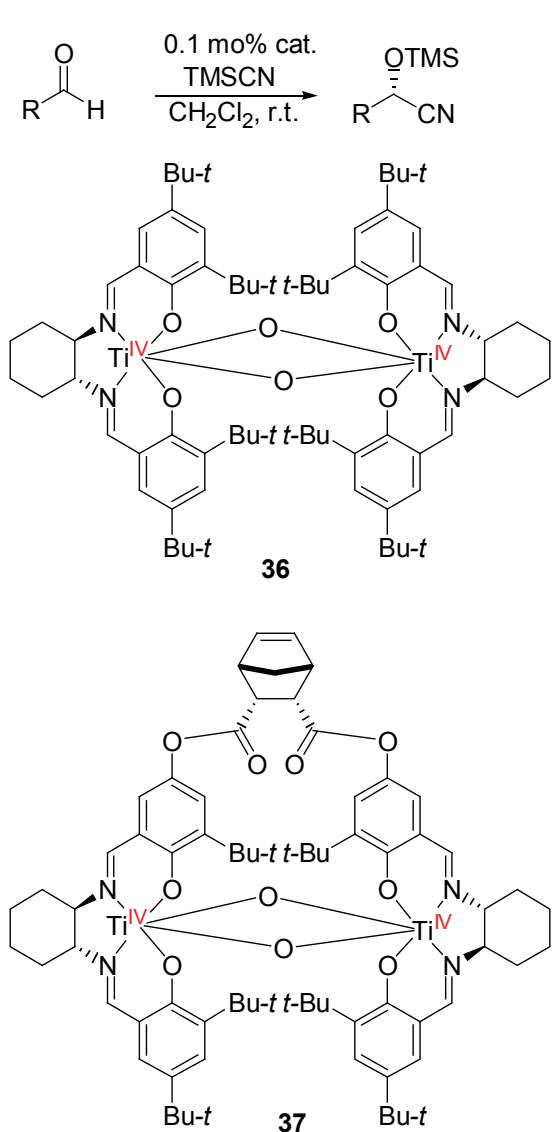

Scheme 13 


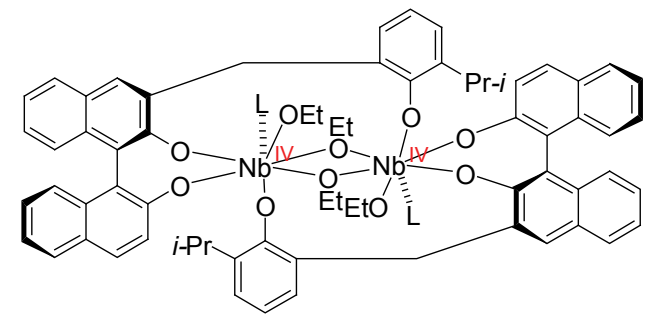

38

图 9 (BINOL-Nb $)_{2}$ 催化剂

Figure 9 (BINOL-Nb $)_{2}$ catalyst

与 Maruoka 双金属钛催化剂有相同的双活化羰基 作用的催化剂还有张万斌小组开发的双噁唑啉醇催化 剂 $39^{[21]}, 40^{[22]}$ 及 Snieckus 开发的 BINOL 衍生的催化剂 41 ${ }^{[23]}$, 这两类多金属锌催化剂都可应用于烷基锌试剂 对醛的亲核反应，且取得了最高为 $99 \%$ 的对映选择性 (图 10).

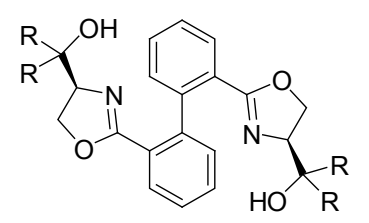

39

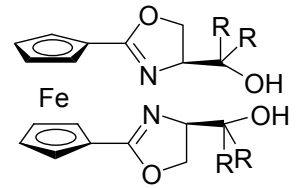

40<smiles>Oc1c(-c2ccc3c(n2)CCCC3)cc2ccccc2c1-c1c(O)c(-c2ccc3c(n2)CCCC3)cc2ccccc12</smiles>

41
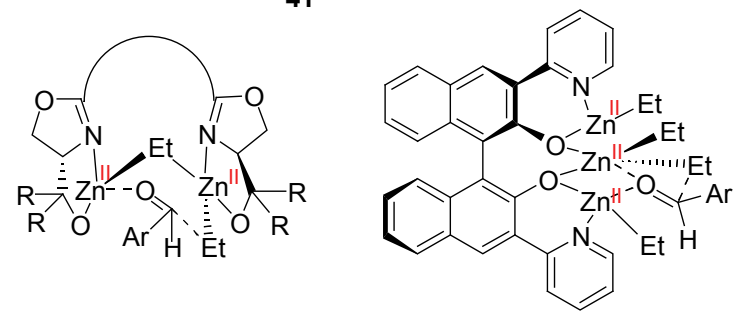

图 10 几种双活化羰基催化剂

Figure 10 Bimetallic catalysts for dueactivation of carbonyl group

氧化偶联反应是一类重要的获得联苯化合物的反 应，该反应可以通过不对称催化得到轴手性联苯二酚化 合物. 开始的研究主要是单金属催化剂配体的设计和反 应条件的寻找. 发展到后来, 化学家们意识到反应机理 是两个相同的自由基的偶联，这正符合双金属催化剂的 催化机理, 于是他们设计了各种手性同核双金属催化 剂, 席夫碱铜双金属催化剂 ${ }^{[24]}$ 和席夫碱钒双金属催化
剂 ${ }^{[25]}$ (图 11). 后者的催化效果非常好，最高达到了 $99 \%$ 的对映选择性，产率也很高，可利用空气中的氧气作为 氧化剂, 催化剂用量也可降低到较低水平.

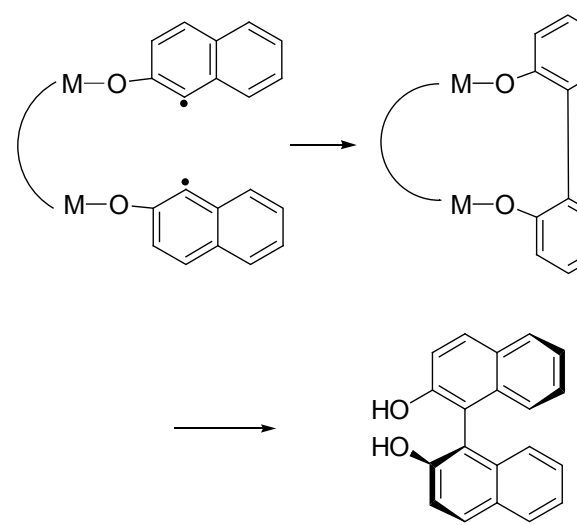

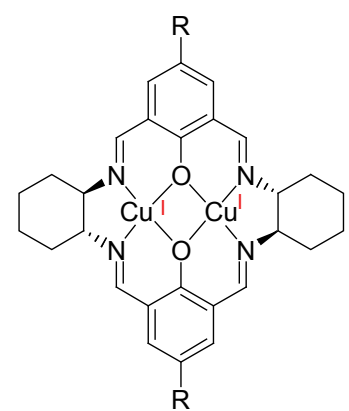

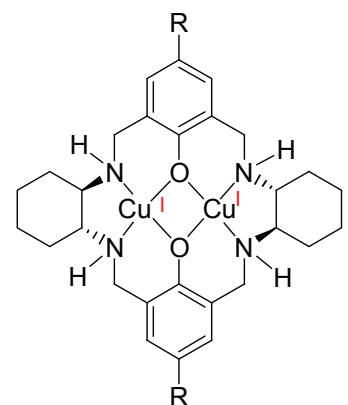

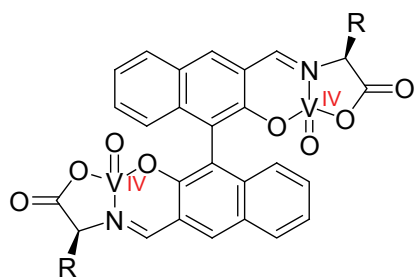

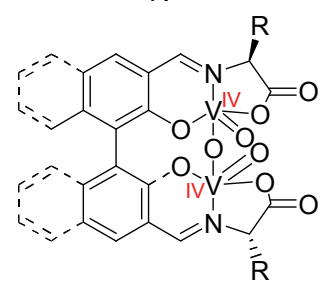

图 11 用于氧化偶联的手性双核同金属催化剂

Figure 11 Chiral bimetallic catalysts for oxidative coupling reaction

最后介绍的是一例手性双金属钯催化剂 FBIP$\mathrm{Cl}^{[26]}$ ，该催化剂用于重排反应时为单金属催化机理，而 用于 $\alpha$-氰基酯对丁烯酩的加成反应时则为双金属催化 机理, 作者通过动力学实验证实了这一点. 由于是双金 属催化机理, 催化剂的活性很高, 该催化剂的用量可以 降低到较低的水平，最低可达 $0.01 \%$, 且此时对映选择 性仍然非常好(Scheme 14).

\section{3 结论}

作为自然界中广泛存在的生命体实现新陈代谢的 主要方式之一, 多金属催化过程已经越来越受到化学家 们的重视. 受到多金属酶的启发，化学家们设计和合成 了大量模拟酶的多核金属催化剂. 本文分别从双核同金 属催化剂和双杂金属催化剂两个方面，介绍了 Shibasaki 

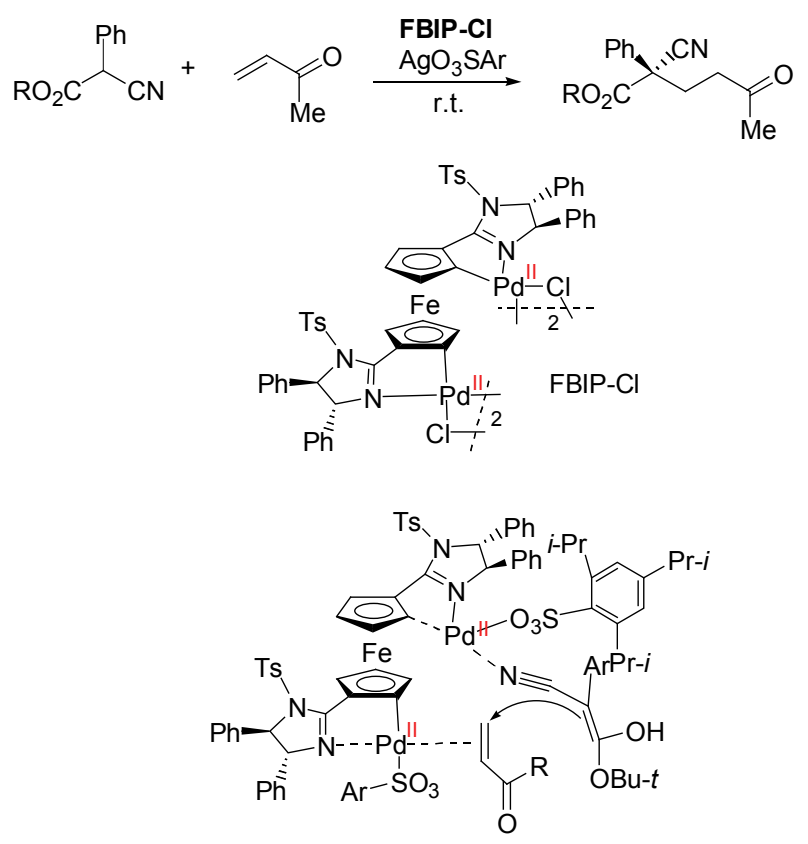

Scheme 15

和 Trost 等课题组开发的双官能手性双金属催化剂以及 Jacobsen 等课题组开发的具有协同作用的双金属催化 剂, 总结了目前双金属催化剂在不对称催化反应中的发 展及应用现状.

目前报道的手性多核金属催化剂中的金属原子之 间多是以氧原子为桥原子或非配位性的链，而氧原子易 与硬的路易酸金属离子结合, 因此现有的多金属催化体 系中使用的金属类型及催化的反应主要是较硬的金属 离子 Lewis 酸催化的反应, 如 1,4-共轭加成反应、Aldol 反应等. 而较软过渡金属催化的金属有机反应作为最有 效的合成方法之一，已经得到了很大的发展，但已知的 金属有机反应大部分为单金属催化过程. 因此, 合理设 计和开发较软过渡金属的手性多核催化剂, 进一步发展 手性双核配体并且开发相应的不对称催化过程, 从而通 过双金属共同作用实现一些单金属无法完成的化学过 程是手性多核金属催化剂未来的发展趋势，化学家们已 经开始进行这方面研究 ${ }^{[27]}$.

\section{References}

[1] Beuken, E. K.; Feringa, B. L. Tetrahedron 1998, 54, 12985.

[2] (a) Shibasaki, M.; Yoshikawa, N. Chem. Rev. 2002, 102, 2187.

(b) Sasai, H.; Tokunaga, T.; Watanabe, S.; Suzuki, T.; Itoh, N.; Shibasaki, M. J. Org. Chem. 1995, 60, 7388 .

(c) Funabashi, K.; Saida, Y.; Kanai, M.; Arai, T.; Sasai, H.; Shibasaki, M. Tetrahedron Lett. 1998, 39, 7557.

(d) Yamada, Y. M. A.; Yoshikawa, N.; Sasai, H.; Shibasaki, M. Angew. Chem., Int. Ed. 1997, 36, 1871.

(e) Nemoto, T.; Ohshima, T.; Shibasaki, M. J. Am. Chem. Soc. 2001, 123, 9474.

(f) Sasai, H.; Arai, S.; Tahara, Y.; Shibasaki, M. J. Org. Chem.
1995, 60, 6656.

(g) Arai, T.; Bougauchi, M.; Sasai, H.; Shibasaki, M. J. Org. Chem. 1996, 61, 2926.

[3] (a) Handa, S.; Gnanadesikan, V.; Matsunaga, S.; Shibasaki, M. J. Am. Chem. Soc. 2007, 129, 4900.

(b) Chen, Z.; Morimoto, H.; Matsunaga, S.; Shibasaki, M. J. Am. Chem. Soc. 2008, 130, 2170.

(c) Hanada, S.; Nagawa, K.; Sohtome, Y.; Matsunage, S.; Shibasaki, M. Angew. Chem., Int. Ed. 2008, 47, 3230.

[4] Yabu, K.; Masumoto, S.; Yamasaki, S.; Hamashima, Y.; Kanai, M.; Du, W.; Curran, D. P.; Shibasaki, M. J. Am. Chem. Soc. 2001, 123, 9908.

[5] Feringa, B. L.; Naasz, R.; Imbos, R.; Arnold, L. A. In Mordern Organocopper Chemistry, Ed.: Krause, N., Wiley-VCH, Weinheim, 2002, p. 224

[6] Feringa, B. L.; Teichert, J. F. Angew. Chem., Int. Ed. 2010, 49, 2486.

[7] Feringa, B. L.; Pineschi, M.; Arnold, L. A.; Imbos, R.; de Vries, A H. M. Angew. Chem., Int. Ed. 1997, 36, 2620.

[8] (a) De Vries, A. H. M.; Meetsma, A.; Feringa, B. L. Angew. Chem., Int. Ed. 1996, 35, 2374.

(b) Arnold, L. A.; Imbos, R.; Mandoli, A.; de Vries, A. H. M.; Naasz, R.; Feringa, B. L. Tetrahedron 2000, 56, 2865.

[9] (a) Feringa, B. L.; Pineschi, M.; Arnold, L. A.; Imbos, R.; de Vries, A. H. M. Angew. Chem., Int. Ed. 1997, 36, 2620.

(b) Feringa, B. L. Acc. Chem. Res. 2000, 33, 346.

[10] (a) Degrado, S. J.; Mizutani, H.; Hoveyda, A. H. J. Am. Chem. Soc. 2001, 123, 755 .

(b) Mizutani, H.; Degrado, S. J.; Hoveyda, A. H. J. Am. Chem. Soc. 2002, 124, 779 .

(c) Hu, A.-G.; Fu, Y.; Xie, J.-H.; Zhou, H.; Wang, L.-X.; Zhou, Q.-L. Angew. Chem., Int. Ed. 2002, 41, 2348.

(c) Alexakis, A.; Polet, D.; Benhaim, C.; Rosset, S. Tetrahedron: Asymmetry 2004, 15, 2199.

(d) Zhang, H.; Fang, F.; Xie, F.; Yang, G.; Zhang, W. Tetrahedron Lett. 2010, 51, 3119.

(e) Yang, B.; Xie, F.; Yu, H.; Shen, K.; Ma, Z.; Zhang, W. Tetrahedron 2011, 67, 6197.

(f) Yu, H.; Xie, F.; Ma, Z.; Liu, Y.; Zhang, W. Adv. Synth. Catal. 2012, 68, 1941.

(g) Shi, M.; Wang, C.; Zhang, W. Chem. Eur. J. 2004, 10, 5507.

(h) Zhang, W.; Shi, M. Synlett 2007, 19.

[11] Stangeland, E. L.; Sammakia, T. Tetrahedron 1997, 53, 16503.

[12] Martin, D.; Kehrli, S.; d'Augustin, M.; Clavier, H.; Mauduit, M.; Alexakis, A. J. Am. Chem. Soc. 2006, 128, 8416.

[13] Zhang, L.; Yang, G.; Shen, C.; Arghib, S.; Zhang, W. Tetrahedron Lett. 2011, 52, 2375.

[14] (a) Trost, B. M.; Ito, H. J. Am. Chem. Soc. 2000, 122, 12003.

(b) Trost, B. M.; Ito, H.; Silcoff, E. R. J. Am. Chem. Soc. 2001, 123, 3367.

(c) Trost, B. M.; Mino, T. J. Am. Chem. Soc. 2003, 125, 2410.

(d) Trost, B. M.; Jaratjaroonphong, J.; Reutrakul, V. J. Am. Chem. Soc. 2006, 128, 2778.

(e) Trost, B. M.; Müller, C. J. Am. Chem. Soc. 2008, 130, 2438

(f) Trost, B. M.; Hitce, J. J. Am. Chem. Soc. 2009, 131, 4572.

[15] (a) Annis, D. A.; Jacobsen, E. N. J. Am. Chem. Soc. 1999, 121, 4147.

(b) Ready, J. M.; Jacobsen, E. N. Angew. Chem., Int. Ed. 2002, 41, 1374.

(c) Mazet, C.; Jacobsen, E. N. Angew. Chem., Int. Ed. 2008, 47, 1762 .

[16] (a) Grachan, M. L.; Tudge, M. T.; Jacobsen, E. N. Angew. Chem., 
Int. Ed. 2008, 47, 1469.

(b) Ruck, R. T.; Jacobsen, E. N. Angew. Chem., Int. Ed. 2003, 42, 4771.

[17] Park, H; Lang, K.; Abboud, K. A.; Hong, S. J. Am. Chem. Soc. 2008, 130, 16484.

[18] (a) Hanawa, H.; Hashimoto, T.; Maruoka, K. J. Am. Chem. Soc. 2003, 125, 1708.

(b) Hanawa, H.; Uraguchi, D.; Konishi, S.; Hashimoto, T.; Maruoka, K. Chem. Eur. J. 2003, 9, 4405.

(c) Kano, T.; Hashimoto, T.; Maruoka, K. J. Am. Chem. Soc. 2005, 127, 11926.

(d) Kano, T.; Hashimoto, T.; Maruoka, K. J. Am. Chem. Soc. 2006, $128,2174$.

[19] (a) Belokon, Y. N.; Caveda-Cepas, S.; Green, B.; Ikonnikov, N. S.; Khrustalev, V. N.; Larichev, V. S.; Moscalenko, M. A.; North, M. J. Am. Chem. Soc. 1999, 121, 3968.

(b) Belokon, Y. N.; Caveda-Cepas, S.; Green, B.; Ikonnikov, N. S.; Khrustalev, V. N.; Larichev, V. S.; Moscalenko, M. A.; North, M. Tetrahedron 2007, 63, 5287.

(c) Zhang, Z.; Wang, Z.; Zhang, R.; Ding, K. Angew. Chem., Int. Ed. 2010, 49, 6746.

[20] Kobayashi, S.; Arai, K.; Shimizu, H.; Ihori, Y.; Ishitani, H.; Yamashita, Y. Angew. Chem., Int. Ed. 2005, 44, 761

[21] Hua, G.; Liu, D.; Xie, F.; Zhang, W. Tetrahedron Lett. 2007, 48, 385.

[22] Imai, Y.; Matsuo, S.; Zhang, W.; Nakatsuji, Y.; Ikeda, I. Synlett 2000, 239.

[23] Milburn, R. R.; Hussain, S. M. S.; Prien, O.; Ahmed, Z.; Snieckus, V. Org. Lett. 2007, 9, 4403.

[24] Gao, J.; Reibenspies, J. H.; Martell, A. E. Angew. Chem., Int. Ed. 2003, 42, 6008.

[25] (a) Luo, Z.; Liu, Q.; Gong, L.; Cui, X.; Mi, A.; Jiang, Y. Chem. Commun. 2002, 914.

(b) Luo, Z.; Liu, Q.; Gong, L.; Cui, X.; Mi, A.; Jiang, Y. Angew. Chem., Int. Ed. 2002, 41, 4532.

(c) Somei, H.; Asano, Y.; Yoshida, T.; Takizawa, S.; Yamataka, H.; Sasai, H. Tetrahedron Lett. 2004, 45, 1841.

[26] (a) Jautze, S.; Peters, R. Angew. Chem., Int. Ed. 2008, 47, 9284. (b) Weber, M.; Jautze, S.; Frey, W.; Peters, R. J. Am. Chem. Soc. 2010, 132, 12222.

[27] For selected cooperative bimetal-catalyzed or mediated reactions: (a) Xi, Z.; Song, Q. J. Org. Chem. 2000, 65, 9157.

(b) Song, Q.; Chen, J.; Jin, X.; Xi, Z. J. Am. Chem. Soc. 2001, 123, 10419 .

(c) Chen, J.; Song, Q.; Wang, C.; Xi, Z. Angew. Chem., Int. Ed. 2001, 40, 1913.

(d) Chen, J.; Song, Q.; Wang, C.; Xi, Z. J. Am. Chem. Soc. 2002, 124, 6238

(e) Tsukada, N.; Mitsuboshi, T.; Setoguchi, H.; Inoue, Y. J. Am. Chem. Soc. 2003, 125, 12102.

(f) Gao, J.; Reibenspies, J. H.; Martell, A. E. Angew. Chem., Int. Ed. 2003, 42, 6008.

(g) Anderson, D. J.; McDonald, R.; Cowie, M. Angew. Chem., Int. Ed. 2007, 41, 3741.

(h) Park, J.; Lang, K.; Abboud, K. A.; Hong, S. J. Am. Chem. Soc. 2008, 130, 16484.

(i) Wu, B.; Gallucci, J. C.; Parquette, J. R.; Rajanbabu, T. V. Angew. Chem., Int. Ed. 2008, 47, 1126.

(j) Zhou, Y.; Xi, Z.; Chen, W. Organometallics 2008, 27, 5911.

(k) Bandini, M.; Eichholzer, A. Angew. Chem., Int. Ed. 2009, 48, 9538.

(1) Liu, L.; Zhang, W.-X.; Wang, C.; Wang, C.; Xi, Z. Angew. Chem., Int. Ed. 2009, 48, 8111.

(m) Rodriguez, B. A.; Delferro, M.; Marks, T. J. J. Am. Chem. Soc. 2009, 131, 5902 .

(n) Deprez, N. R.; Sanford, M. S. J. Am. Chem. Soc. 2009, 131, 11239.

(o) Powers, D. C.; Ritter, T. Nat. Chem. 2009, 1, 302.

(p) Sachse, A.; John, M.; Meyer, F. Angew. Chem., Int. Ed. 2010, 49, 1986.

(q) Endo, K.; Ogawa, M.; Shibata, T. Angew. Chem., Int. Ed. 2010, 49, 2410.

(r) Zhu, C.; Yukimura, N.; Yamane, M. Organometallics 2010, 29, 2098.

(s) Velian, A.; Lin, S.; Miller, A. J. M.; Day, M. W.; Agapie, T. J. Am. Chem. Soc. 2010, 132, 6296.

(Zhao, X.) 